\title{
Dissecting the cosmic infrared background with 3D instruments (Research Note)
}

\author{
D. L. Clements ${ }^{1}$, K. G. Isaak ${ }^{2}$, S. C. Madden ${ }^{3}$, and C. Pearson ${ }^{4,5}$ \\ 1 Astrophysics Group, Blackett Laboratory, Imperial College, Prince Consort Road, London SW7 2BW, UK \\ e-mail: d.clements@imperial.ac.uk \\ 2 School of Physics and Astronomy, Cardiff University, The Parade, Cardiff CF24 3AA, UK \\ e-mail: kate.isaak@astro.cf.ac.uk \\ 3 Service d'Astrophysique, Bât. 609, Orme des Merisers, CEA Saclay, 91191 Gif-sur-Yvette, France \\ e-mail: madden@cea.fr \\ 4 Institute of Space and Astronautical Science, Japan Aerospace Exploration Agency, Yoshinodai 3-1-1, Sagamihara, \\ Kanagawa 229-8510, Japan \\ e-mail: cpp@ir.isas.jaxa.jp \\ 5 ISO Data Centre, European Space Agency, Villafranca del Castillo, PO Box 50727, 28080 Madrid, Spain
}

Received 8 November 2006 / Accepted 21 December 2006

\begin{abstract}
Context. The cosmic infrared background (CIB) consists of emission from distant, dusty, star-forming galaxies. Energetically, the $\mathrm{CIB}$ is very important as it contains as much energy as the extragalactic optical background. The nature and evolutionary status of the objects making up the background are, however, unclear.

Aims. The CIB peaks at $\sim 150 \mu \mathrm{m}$, and as such is most effectively studied from space. The limited apertures of space-borne telescopes set the angular resolution that can be attained, and so even Herschel, with its $3.5 \mathrm{~m}$ diameter, will be confusion-limited at this wavelength at $\sim 5 \mathrm{mJy}$. The bulk of the galaxies contributing to the CIB are fainter than this, so it is difficult to study them without interferometry. Here we present the results of a preliminary study of an alternative way of probing fainter than the continuum confusion limit using far-IR imaging spectroscopy. An instrument capable of such observations is being planned for SPICA - a proposed Japanese mission with an aperture equivalent to that of Herschel and more than 2 orders of magnitude more sensitive.

Methods. In this paper we investigate the potential of imaging spectrometers to break the continuum confusion limit. We have simulated the capabilities of a spectrometer with modest field of view $\left(2^{\prime} \times 2^{\prime}\right)$, moderate spectral resolution $(R \sim 1-2000)$ and high sensitivity.

Results. We find that such an instrument is capable of not only detecting line emission from sources with continuum fluxes substantially below the confusion limit, but also of determining their redshifts and, where multiple lines are detected, some emission line diagnostics.

Conclusions. 3-D imaging spectrometers on cooled far-IR space telescopes will be powerful new tools for extragalactic far-IR astronomy.
\end{abstract}

Key words. galaxies: high-redshift - infrared: galaxies - galaxies: starburst - instrumentation: spectrographs space vehicles: instruments

\section{Introduction}

The discovery of the Cosmic Infrared Background (CIB) (Puget et al. 1996; Fixsen et al. 1998) provided a key step in our understanding of the history of the universe. The energy in the CIB is comparable to that in the integrated UV/optical/near-IR (UVOIR) background. Since the CIB comes from obscured systems and the UVOIR from unobscured systems this means that roughly $50 \%$ of energy produced in the history of the Universe comes from obscured systems. The nature of the objects making up the CIB, however, remains unclear. Source populations that contribute significantly to the CIB have been found in submillimetre surveys (Blain et al. 2002, and references therein), in the far-IR (Puget et al. 1999), and in the mid-IR (Dole et al. 2006), with as much as $70 \%$ (Dole et al. 2006) of the CIB now believed to have been resolved into individual sources. Much of this work, however, has been done at wavelengths far away from the $150 \mu \mathrm{m}$ peak of the CIB, and is reliant on extrapolations.

The Herschel far-IR mission (Pilbratt 2003), to be launched in 2008, will be capable of deep observations around the peak of the CIB, and will be sensitive enough to resolve a significant fraction of CIB sources at wavelengths where their spectral energy distributions (SEDs) peak (Dole et al. 2004). With a $3.5 \mathrm{~m}$-diameter mirror, Herschel will have the largest satellite primary to date, but even this will only give an angular resolution of $\sim 10^{\prime \prime}$ at $150 \mu \mathrm{m}$. The source density of CIB sources on the sky is such that confusion, rather than instrument sensitivity, is the main limitation on the faintness at which an individual CIB source can be detected - for exmple, the confusion limit of the PACS instrument on Herschel (Poglitsch et al. 2005) has been estimated to be $\sim 5 \mathrm{mJy}$ at the $\sim 150 \mu \mathrm{m}$ peak of the CIB (Jeong et al. 2006). Extrapolations from the deep SPITZER 
surveys suggest that Herschel will therefore only be able to resolve $\sim 50 \%$ of the CIB (Dole et al. 2004) at best, even at its shortest operating wavelength.

There are of course ways in which one can probe below the confusion limit by using observations at other wavelengths or through statistical approaches. This includes extraction of long wavelength fluxes at the positions of sources detected at shorter wavelengths which has proved successful in some studies (e.g. Dole et al. 2006). There remains the worry that such studies can be biased against redder sources, which may be missing from the short wavelength catalogs but still contribute strongly at long wavelengths. Such issues can only be avoided by detecting sources at the long, CIB, wavelengths themselves which will require observations below the continuum confusion. In this research note we put forward the results of work that has been done to use an additional observational dimension to break the confusion limit - that of spectroscopy. We will demonstrate that through spectroscopy one can identify sources that lie significantly below the traditional (contiuum) confusion limit, and thus to probe a population that contributes significantly to the CIB, that cannot presently be studied in any other way.

\section{Modeling blank field far-IR line surveys}

\subsection{A far-IR imaging spectrometer}

The key feature of an instrument designed to conduct blank field searches for emission lines is that it must simultaneously cover a significant field of view over a significant spectral range with sufficient sensitivity to detect the lines against the continuum. Imaging spectroscopy at far-infrared, infrared and optical wavelengths can be achieved using a number of different instrumental techniques, including imaging fourier transform spectroscopy as employed in the Herschel SPIRE instrument (Griffin et al. 2004), integral field spectroscopy using a grating and image slicer - as employed in the Herschel PACS instrument (Poglitsch et al. 2005), using imaging Fabry-Perots - as employed in SPIFI (Stacey et al. 2002), and at the longer wavelengths with heterodyne arrays using ultra-sensitive mixers - as employed in HARP-B (Smith et al. 2003). Each technique is more or less appropriate to a particular application and a particular wavelength range/instantaneous bandwidth requirement.

Instrument concepts based on the first two of these techniques are currently under consideration for an imaging spectrometer (ESI, the European SPICA instrument) for the $35-210 \mu \mathrm{m}$ waveband for SPICA (Swinyard et al. 2006). For the purpose of this paper our considerations of the instrument itself are restricted to its deliverables, namely a data cube with a particular two-dimensional spatial extent and angular resolution and a given spectral range and resolution in the third (spectral) direction, with a specified sensitivity in each spectral channel. We have based our simulations on what we believe to be feasible for a cooled $(4 \mathrm{~K}), 3.5 \mathrm{~m}$-diameter primary, telescope such as SPICA: a spatial resolution of $8^{\prime \prime}$ as set by the diffraction limit at $\sim 120 \mu \mathrm{m}$; an instantaneous spectral coverage of 60 to $210 \mu \mathrm{m}$ and a fixed channel width of $\Delta \lambda \approx 0.176 \mu \mathrm{m}$, resulting in a spectral resolution ranging from 350 at $60 \mu \mathrm{m}$ to 1200 at $210 \mu \mathrm{m}$ and an instantaneous field of view of $128^{\prime \prime} \times 128^{\prime \prime}$. These instrumental parameters are very similar to those expected in the two longest wavelength channels of the ESI far-infrared instrument concept.
Table 1. Galaxies used to provide far-IR lines for templates.

\begin{tabular}{cc}
\hline \hline Continuum Template & Line Template \\
\hline Normal cold & Maffei 2 \\
Normal & Maffei 2 \\
Starburst M 82 & M 82 \\
Starburst & M 82 \\
$10^{11} L_{\odot}$ LIG & NGC 253 \\
$10^{11.5} L_{\odot}$ LIG & NGC 253 \\
Cold LIG & NGC 253 \\
Hot ULIRG & Arp 220 \\
Cold ULIRG & Arp 220 \\
Sy1 & No lines \\
Sy2 & No lines \\
\hline
\end{tabular}

\subsection{The far-IR sky}

To assess the ability of our far-IR imaging spectrometer to detect line-emitting sources in the CIB, we must model the far-IR sky. Our starting place is the current generation of empirical number count models that fit all current constraints on galaxy counts from optical to submm, including the strength of the CIB. The model we have chosen to adopt is the burst mode evolution model of Pearson (2006) since this provides all the necessary features we require, and, in later work, can easily be compared and contrasted with Pearson's parallel bright galaxy evolution model. The model we use is based on an evolving, type-dependent, far-infrared luminosity function which makes use of a set of 11 optical-to-submm template SEDs. The template objects range from normal, Milky-Way type galaxies, through increasingly far-IR luminous objects to extreme ultraluminous infrared galaxies (ULIRGs) and type 1 and 2 AGNs. To simulate a region of the far-IR sky we begin with a simulated catalogue of sources with far-infrared fluxes, luminosities, redshifts and realistic SEDs taken from the galaxy evolution model. Each source in the catalogue is parameterised by a wavelength and a flux, which are assigned based on the SED template type. At this point, the template fluxes are continuum only. Far-IR emission (and absorption) lines are added to these templates using line strengths derived from LWS far-IR spectroscopy for a range of nearby galaxies (e.g. Negishi et al. 2001), as tabulated in Table 1. The resulting templates, including lines, are shown in Fig. 1. We only include the brightest far-IR lines, and no MIR lines, at this stage since these will be the most useful for source detection in the far-IR.

To generate the data cube that will come from observing this catalog we compile a list of sources in our field of view. The integrated flux contributed to each 8 " pixel by all the objects that lie within it is then calculated. The template spectrum for each object contributing to a spatial pixel is shifted in wavelength by the source redshift and then normalised to the flux of the source at the fiducial catalog wavelength $(40 \mu \mathrm{m}$, the wavelength at which the original catalog was normalised). The flux at a given, observed wavelength is then interpolated from the template and added to the appropriate wavelength channel in the data cube. This process is repeated for each $8^{\prime \prime} \times 8^{\prime \prime}$ spatial pixel to build up the $16 \times 16$ spatial cube, each pixel made up of a 3 rd dimension of 852 spectral channels. Random Gaussian noise is then added to each of these channels at a level appropriate to the instrument sensitivity being simulated.

At the same time as the data cube is generated, we also produce a "truth catalog" for the field which is comprised of $120 \mu \mathrm{m}$ fluxes (to allow comparison with the sensitivity of current and future instruments such as PACS and Spittzer), 


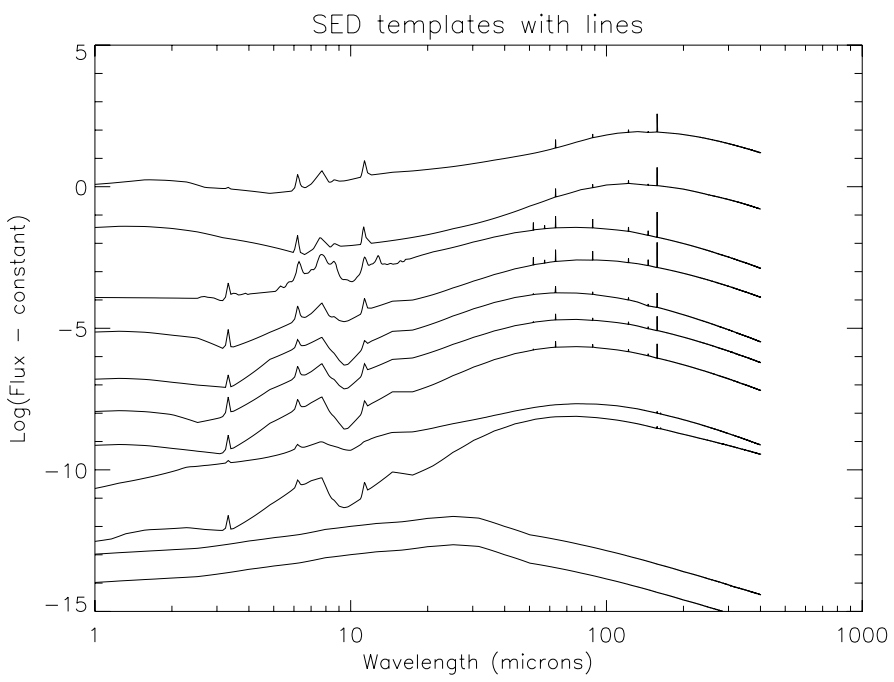

Fig. 1. Spectral energy distributions used for the models. From the top downwards these SEDs correspond to the following SED types in the models: normal cold, normal, starburst M 82, starburst, $10^{11} L_{\mathrm{FIR}}$ LIG, $10^{11.5} L_{\mathrm{FIR}}$ LIG, Cold LIG, Hot ULIRG, Cold ULIRG, Sy1, Sy2.

redshifts, positions and source classifications for objects in the $128^{\prime \prime} \times 128^{\prime \prime}$ field of view. This truth catalogue is also used to calculate the conventional " 20 beams per source" confusion limit which, for this field, is $4.3 \mathrm{mJy}$.

\section{Results of models}

To determine the completeness of source identification via farIR line emission, we search the simulated data cube for sources with strong line emission; we then compare the resulting list of sources with the "truth catalog" derived from the original simulations. Line detection is conducted in the following way: at each image pixel the continuum is removed by fitting and subtracting a 4 th order polynomial; the standard deviation, $\sigma$, of the resulting continuum-subtracted spectrum is then calculated; a source is defined to contain a line if there exist channels in the spectrum in which the line flux is greater than some pre-defined multiple of this rms noise, i.e. $n \sigma$. We find through comparison to the "truth catalog" that a reliable, i.e. no false detections, list of line emitting sources is produced when $n=4.5$, i.e. when lines with significance $\geq 4.5 \sigma$ are selected.

Figure 2 compares the broad band image of the field at $120 \mu \mathrm{m}$ (left) with a continuum-subtracted image at a wavelength matching the faintest detected line-emitting source, at a wavelength of $\sim 209 \mu \mathrm{m}$ (right), for a simulation with $1 \sigma$ noise of $0.4 \mathrm{mJy}$. We note that this line-emitting source has a continuum flux of $0.56 \mathrm{mJy}$, nearly an order of magnitude fainter than the traditional confusion limit. However it is still significantly detected through its line emission. The number of sources recovered through their line emission as a function of continuum flux, compared to the initial distribution of fluxes in the input catalog is shown in Fig. 3. This clearly demonstrates the power of the technique, in that we recover all sources above the conventional confusion limit and a substantial fraction of those with fluxes up a factor of 10 below it. As expected, the fraction detected drops as the source gets fainter. The potential for breaking the continuum confusion limit through the detection of sources in their far-IR line emission is clear.

It is possible not only to locate a source in RA-Dec via blankline surveys but also, very importantly, to determine its redshift.
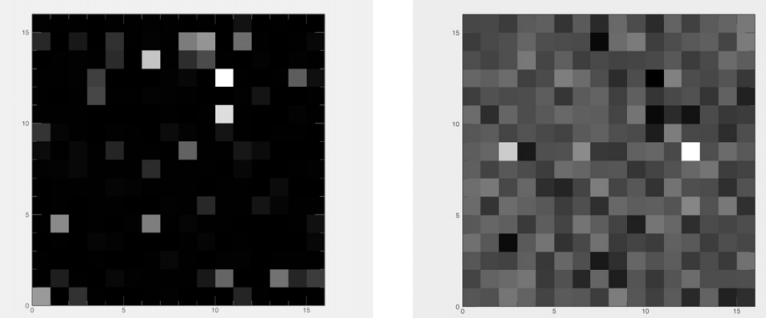

Fig. 2. Source detected in line below confusion limit. Left: continuum $120 \mu \mathrm{m}$ image of the field. Right: narrow band image of the field at the wavelength at which a $560 \mu \mathrm{Jy} 120 \mu \mathrm{m}$ continuum flux source is detected by its line emission. Note that the source is clearly detected in the line image (the rightmost bright pixel, at location $(12,8)$ ) while in the continuum image nothing can be seen because of the confusion noise. Greyscales not the same, but chosen to make this effect clear. The additional source seen to the left in the line image is below our $4.5 \sigma$ detection threshold.

This requires several assumptions to be made. In some cases we detect more than one emission line in a given object, making redshift determination quite simple. In most cases, however, only one line is detected: in such cases the single lines detected are usually one of two bright far-IR emission lines [CII] $157.7 \mu \mathrm{m}$ or [OI] $63.2 \mu \mathrm{m}$. In a typical dust SED which is characteristic of dust at a temperature of $\sim 35 \mathrm{~K}$, the [CII] line lies longward of the SED peak, while [OI] lies at shorter wavelengths. If we assume that the dominant contribution to the confused continuum emission comes from the line emitting source, then by considering the position of the line relative to the SED peak it is possible to guess which far-IR species is responsible for the emission line and thus get an accurate determination of redshift. The efficacy of this is shown in Fig. 3, where the inferred redshifts of detected sources with $S_{120 \mu \mathrm{m}}>1 \mathrm{mJy}$ are compared to the catalogue as a whole. As can be seen, we recover $100 \%$ of the redshifts for sources at $z<2.5$ that are brighter than $1 \mathrm{mJy}$ i.e. $\sim 5$ times fainter than the conventional confusion limit. By $z \sim 2.5$, both of the two strongest FIR lines, [CII] $157.7 \mu \mathrm{m}$ and [OI]63.2 $\mu \mathrm{m}$ have moved out of the passband of our simulated instrument. We therefore do not expect to obtain any of these higher redshifts in the current simulation.

We thus find that blind, blank-field spectral surveys are capable not only of detecting sources well below the confusion limit but of obtaining their redshifts at the same time. Early analysis suggests we can distinguish between different luminosity evolution models (the burst and bright models from Pearson 2006) with a survey $10^{\prime} \times 10^{\prime}$ or less in size using these techniques. This is something not possible using broadband number counts. Operation at higher redshifts will come from additional narrow lines not in the current model, such as the strong [SiII] $34.8 \mu \mathrm{m}$, [SIII] 33.5 $\mu \mathrm{m}$ and [OIV] $24.9 \mu \mathrm{m}$ lines, some of which can be used as emission line diagnostics. Template correlation working on redshifted PAH features will also allow us to probe high redshifts.

\section{Conclusions}

We have examined the potential for of imaging spectroscopic instruments in studying the sources responsible for the CIB. The main limiting factor in CIB studies for far-IR missions is confusion resulting from the high continuum source density and 

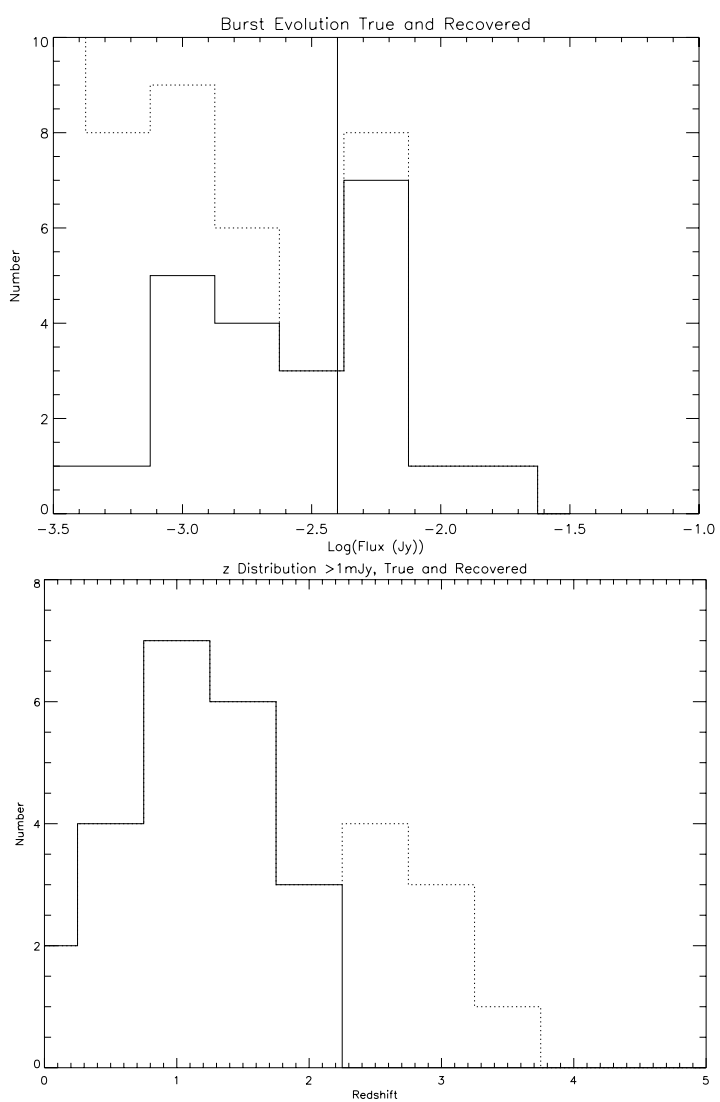

Fig. 3. Recovery of Objects, with redshifts, from the FIR data cube. Top: shows the input flux distribution at $120 \mu \mathrm{m}$ from the "truth catalog" (dotted line) and the sources detected from their line emission as a function of their $120 \mu \mathrm{m}$ continuum flux (solid line) for a $4.5 \sigma$ line detection threshold and a $1 \sigma$ noise of 0.4 mJy per channel. The conventional continuum confusion limit of 20 beams per source is shown as a vertical line. Note that many sources below this confusion limit are detected, with sources having continuum fluxes nearly an order of magnitude below the limit being accessible. Bottom: the redshift distribution from the "truth catalog" for sources brighter than $1 \mathrm{mJy}$ at $120 \mu \mathrm{m}$ (dotted line) and the redshift distribution for sources brighter than $1 \mathrm{mJy}$ at $120 \mu \mathrm{m}$ recovered from the $4.5 \sigma$ line detected sources. Note that all sources with $z<2.5$ and $F_{120}>1$ mJy have been recovered. large instrumental beams. The use of imaging spectrometers enables the detection of sources by their line rather than continuum emission, allowing us to detect sources whose continuum flux is up to an order of magnitude below the conventional confusion limit. Furthermore, a by-product of the analysis of the line and continuum emission of sources detected in this way can be used to determine source redshifts. We believe there is considerable mileage in deep, blank-field spectral line surveys in the far-infrared and will discuss the results of more extensive modeling in a future, more substantial paper.

Acknowledgements. This work is supported in part by PPARC, CNES, the French Programmes Nationaux and ESA, and is a product of the SPICA-ESI Phase A study. Our thanks go to all the other members of the ESI and SPICA teams, and to the anonymous referee for many helpful comments.

\section{References}

Blain, A. W., Smail, I., Ivison, R. J., Kneib, J.-P., \& Frayer, D. T. 2002, PhR., 369, 111

Dole, H., Rieke, G. H., Lagache, G., et al. 2004, ApJS, 154, 93

Dole, H., Lagache, G., Puget, J.-L., et al. 2006, A\&A, 451, 417

Fixsen, D. J., Dwek, E., Mather, J. C., Bennett, C. L., \& Shafer, R. A. 1998, ApJ, 508,123

Griffin, M. L., Swinyard, B. M., \& Vigroux, L. 2004, Optical, Infrared, and Millimeter Space Telescopes, ed. J. C. Mather, Proc. SPIE, 5487, 413

Jeong, W.-S., Pearson, C. P., Lee, H. M., Pak, S. J., \& Nakagawa, T. 2006, MNRAS, 369, 281

Negishi, T., Onaka, T., Chan, K.-W., \& Roellig, T. L. 2001, A\&A, 375, 566

Pearson, C. 2006, in preperation

Pilbratt, G. L. 2003, IR Space Telescopes and Instruments, ed. J. C. Mather, Proc. SPIE, 4850, 586

Poglitsch, A., Waelkens, C., Geis, N., et al. 2005, Astron. Nachr., 326, 583

Puget, J.-L., Abergel, A., Bernard, J.-P., et al. 1996, A\&A, 308, L5

Puget, J.-L., Lagache, G., Clements, D. L., et al. 1999, A\&A, 345, 29

Smith, H., et al. 2003, Millimeter and Submillimeter Detectors for Astronomy, ed. T. G. Phillips, \& J. Zmuidzinas, Proc. SPIE, 4855, 338

Stacey, G., et al. 2002, EAS Publications Series, Volume 4, Proceedings of Infrared and Submillimeter Space Astronomy, held 11-13 June, 2001, ed. M. Giard, J. P. Bernanrd, A. Klotz, \& I. Ristorcelli (EDP Sciences), 419

Swinyard, B., et al. 2006, Proc. SPIE, in press 